\title{
Change of numeraire in the two-marginals martingale transport problem
}

\author{
Luciano Campi ${ }^{1}$. Ismail Laachir ${ }^{2}$. \\ Claude Martini ${ }^{2}$
}

Received: 1 March 2016 / Accepted: 17 September 2016 / Published online: 28 December 2016

(C) The Author(s) 2016. This article is published with open access at Springerlink.com

\begin{abstract}
In this paper, we apply change of numeraire techniques to the optimal transport approach for computing model-free prices of derivatives in a two-period setting. In particular, we consider the optimal transport plan constructed in Hobson and Klimmek (Finance Stoch. 19:189-214, 2015) as well as the one introduced in Beiglböck and Juillet (Ann. Probab. 44:42-106, 2016) and further studied in HenryLabordère and Touzi (Finance Stoch. 20:635-668, 2016). We show that in the case of positive martingales, a suitable change of numeraire applied to Hobson and Klimmek (Finance Stoch. 19:189-214, 2015) exchanges forward start straddles of type I and type II, so that the optimal transport plan in the subhedging problems is the same for both types of options. Moreover, for Henry-Labordère and Touzi's (Finance Stoch. 20:635-668, 2016) construction, the right-monotone transference plan can be viewed as a mirror coupling of its left counterpart under the change of numeraire.
\end{abstract}

Keywords Robust hedging · Model-independent pricing · Model uncertainty · Optimal transport · Change of numeraire $\cdot$ Forward start straddle

Mathematics Subject Classification (2010) $91 \mathrm{G} 20 \cdot 91 \mathrm{G} 80$

\section{JEL Classification G13}

\section{Campi}

1.campi@1se.ac.uk

I. Laachir

ilaachir@zeliade.com

C. Martini

cmartini@zeliade.com

1 Department of Statistics, Columbia House, London School of Economics and Political Sciences, Houghton Street, London WC2A 2AE, UK

2 Zeliade Systems, 56 Rue Jean-Jacques Rousseau, 75001 Paris, France 


\section{Introduction}

Let $\mu$ and $v$ be two probability measures on the positive half-line $\mathbb{R}_{++}:=(0, \infty)$, both with unit mean and satisfying $\mu \preccurlyeq v$ in the sense of the convex order, meaning that $\int f d \mu \leq \int f d \nu$ for all convex functions $f: \mathbb{R}_{++} \rightarrow \mathbb{R}$. A classical theorem by Strassen [16] shows the existence of a martingale $M=\left(M_{t}\right)_{t=0}^{2}=(1, X, Y)$ in discrete time with $X \sim \mu$ and $Y \sim v$. Let $\mathcal{M}(\mu, v)$ denote the set of all possible laws for such discrete martingales with pre-specified marginals $\mu, v$. If we interpret the process $M$ as a price of a given stock, any function $C(x, y)$ can be seen as a path-dependent option written on that stock.

Motivated by the issue of model uncertainty (see e.g. the seminal paper [8] and the survey [9]), there has recently been a flourishing of articles on the problem of finding a model-free upper (resp. lower) bound for the price of a given option $C$, which consists in maximizing (resp. minimizing) the expectation $\mathbb{E}^{Q}[C(X, Y)]$ over all measures $Q \in \mathcal{M}(\mu, v)$. Indeed, any such measure $Q$ corresponds to some model for the price process of the underlying. In the model-free setting, such a price process is required to be a martingale (hence free of arbitrage) and to have pre-specified marginals $\mu$ and $\nu$, which can be deduced as usual from the observation of European call option prices via the Breeden-Litzenberger formula. Therefore, in this context $\mathcal{M}(\mu, v)$ is the set of risk-neutral pricing measures which are compatible with the observed call option prices. The upper bound $\sup _{Q \in \mathcal{M}(\mu, v)} \mathbb{E}^{Q}[C(X, Y)]$, for instance, corresponds essentially to the cost of the least expensive semi-static strategy that superreplicates the given payoff. The lower bound has an analogous interpretation as subreplication price.

These optimization problems have been recently tackled using an approach based on optimal transport (see e.g. the papers [1-5, 7, 10], among others). More specifically, Beiglböck and Juillet [1] perform a thorough analysis of martingale transport problems and, among other results, prove that for a certain class of payoffs, the optimal probabilities are of special type, called the left-monotone and right-monotone transference plans. Later on, Henry-Labordère and Touzi [7] provide an explicit construction of such optimal transference plans for a more general class of payoffs $C$ that satisfy the so-called generalized Spence-Mirrlees condition

$$
C_{x y y}>0 .
$$

Finally, Hobson and Klimmek [10] consider forward start straddles of type II, whose payoff is $|Y-\alpha X|$ when the strike is $\alpha$, while we recall for later use that the payoff of a forward start straddle of type I is given by $\left|\frac{Y}{X}-\alpha\right|$. In the case $\alpha=1$, the authors construct another optimal transference plan giving the model-free subreplication price of a forward start straddle of type II, whose payoff does not satisfy the condition (1.1) above.

In this paper, we study the effect of a change of numeraire on the martingale optimal transport approach to model-free pricing. To our knowledge, change of numeraire has never been used so far in connection with optimal transport and robust pricing. We focus on the optimal transference plans mentioned above in the case of marginals whose support is $\mathbb{R}_{++}$, i.e., we consider positive martingales with given marginals. Our main results can be briefly stated as follows. Regarding the Hobson and Klimmek 
[10] optimal coupling measure, it turns out that the change of numeraire exchanges forward start straddles of type I and type II with strike 1. As consequence, this yields that the optimal transport plan in the subhedging problems is the same for both types of forward start straddles. This complements, using a different method, the results in Hobson and Klimmek [10] on forward start straddles of type II. On the other hand, regarding the Beiglböck and Juillet [1] and Henry-Labordère and Touzi [7] left- and right-monotone optimal transport plans, the change of numeraire can be viewed as a mirror coupling for positive martingales. More precisely, we show that the rightmonotone transport plan can be obtained with no effort from its left-monotone counterpart by suitably changing numeraire. The effect of such a transformation on the generalized Spence-Mirrlees condition is also studied. Other invariance properties by change of numeraire are also proved along the way. An extended version of the present paper can be found in Laachir's PhD thesis [14, Chap. 1].

The paper is structured as follows. We introduce in Sect. 2 the change of numeraire and prove its main properties. In Sect. 3, we consider forward start straddles and extend the results in [10] to forward start straddles of type I. In Sect. 4, we give an application of change of numeraire to left- and right-monotone transference plans for positive martingales.

\section{Notations:}

- Let $X$ be any random variable defined on some measurable space $(\Omega, \mathcal{F})$. We denote by $\mathcal{L}_{Q}(X)$ the law of $X$ under some measure $Q$. For the expectation of $X$ under $Q$, we use the notation $\mathbb{E}^{Q}[X]$.

- We use $\mathcal{P}=\mathcal{P}\left(\mathbb{R}_{++}\right)$for the set of all probability measures $\mu$ on $\mathbb{R}_{++}:=(0, \infty)$, equipped with the Borel $\sigma$-field $\mathcal{B}\left(\mathbb{R}_{++}\right)$, and set

$$
\mathcal{P}_{1}=\mathcal{P}_{1}\left(\mathbb{R}_{++}\right):=\left\{\mu \in \mathcal{P}: \int_{\mathbb{R}_{++}} x \mu(d x)=1\right\} .
$$

The subset of all measures $\mu \in \mathcal{P}_{1}$ having a positive density, say $p_{\mu}$, with respect to Lebesgue measure is denoted by $\mathcal{P}_{1}^{d}$.

- If $\mu, v \in \mathcal{P}_{1}$, then $F_{\mu}, F_{\nu}$ denote their respective cumulative distribution functions. We also use the notation $\delta F$ for the difference between the two, i.e.,

$$
\delta F=F_{\nu}-F_{\mu} .
$$

- For any function $x \mapsto q(x)$, we use the notation $\bar{q}(x):=1-q(x)$, and we denote by $G_{\mu}(x):=\int_{(0, x)} y \mu(d y), x>0$, the cumulated expectation of any measure $\mu$. Finally, Id denotes the identity function.

\section{Change of numeraire}

The technique of change of numeraire was first introduced by Jamshidian [12] in the context of interest rate models and turned out to be a very powerful tool in derivatives pricing (see Geman et al. [6], Jeanblanc et al. [13, Sect. 2.4] and the other references 
therein for further details). Here we show that such techniques can be fruitfully transposed to a model-free setting.

We consider a two-period financial market with one riskless asset, whose price is identically equal to one, and one risky asset whose discounted price evolution is modelled by the process $\left(M_{t}\right)_{t=0}^{2}=(1, X, Y)$. The random variables $X$ and $Y$, modelling respectively the prices at time $t=1$ and $t=2$, are defined on the canonical measurable space $(\Omega, \mathcal{F})$, where $\Omega=\Omega_{1} \times \Omega_{2}$ with $\Omega_{1}=\Omega_{2}=\mathbb{R}_{++}$and $\mathcal{F}=\mathcal{B}(\Omega)$. For any $\omega=\left(\omega_{1}, \omega_{2}\right) \in \Omega$, we set $X(\omega)=\omega_{1}$ and $Y(\omega)=\omega_{2}$. This space is equipped with the filtration $\mathbb{F}=\left(\mathcal{F}_{t}\right)_{t=0}^{2}$, where $\mathcal{F}_{0}=\{\emptyset, \Omega\}, \mathcal{F}_{1}=\sigma(X)$ and $\mathcal{F}_{2}=\sigma(X, Y)$. The martingale property always refers to this filtration. The final ingredients of our setting are the two marginal laws $\mu$ and $\nu$, which are probability measures on $\left(\Omega_{1}, \mathcal{B}\left(\mathbb{R}_{++}\right)\right)$and $\left(\Omega_{2}, \mathcal{B}\left(\mathbb{R}_{++}\right)\right)$, so that $X$ (resp. $Y$ ) has law $\mu$ (resp. v). Throughout the whole paper, we work under the following standing assumption:

Assumption 2.1 The marginals $\mu$ and $v$ have unit mean and satisfy $\mu \preccurlyeq v$ in the sense of the convex order, i.e., $\int f d \mu \leq \int f d v$ for all convex functions $f: \mathbb{R}_{++} \rightarrow \mathbb{R}$.

Let $\mathcal{M}(\mu, v)$ denote the set of all probability measures on $(\Omega, \mathcal{F})$ such that $X \sim \mu$, $Y \sim \nu$, and $M$ is a martingale. As already claimed in the introduction, by a classical theorem in [16], we know that Assumption 2.1 guarantees that $\mathcal{M}(\mu, v)$ is nonempty.

\subsection{The one-dimensional symmetry operator $S$}

As a preliminary step, we first consider the change of numeraire in a static setting, i.e., for the marginal laws. Thus, we define the (marginal) symmetry operator $S$ as an operator acting on the space of probability measures on $\left(\mathbb{R}_{++}, \mathcal{B}\left(\mathbb{R}_{++}\right)\right)$by

$$
S(\mu):=\mathcal{L}_{\bar{\mu}}(1 / X), \quad \mu \in \mathcal{P}\left(\mathbb{R}_{++}\right),
$$

where $\bar{\mu}$ is the probability measure defined by $\bar{\mu}(A)=\mathbb{E}^{\mu}\left[X \mathbf{1}_{A}\right]$ for any $A \in \mathcal{B}\left(\mathbb{R}_{++}\right)$.

Remark 2.2 Financially speaking, $S(\mu)$ is the law of the riskless asset price at time $t=1$ measured in units of the risky one under the new probability $X d \mu$. This is the usual change of measure associated to a change of numeraire. An analogous interpretation applies to $S(v)$.

Notice that if $\mu \in \mathcal{P}_{1}$, i.e., it has unit mean, then $S(\mu) \in \mathcal{P}_{1}$, too, due to the equalities $\mathbb{E}^{S(\mu)}[X]=\mathbb{E}^{\mu}[X / X]=1$. In the case where $\mu \in \mathcal{P}_{1}^{d}$ with density $p_{\mu}$, the new measure $S(\mu)$ has a density, too, and this is given by

$$
p_{S(\mu)}(x)=\frac{p_{\mu}(1 / x)}{x^{3}}, \quad x>0 ;
$$

hence in particular we have $S(\mu) \in \mathcal{P}_{1}^{d}$. Moreover, $S$ is an involution, i.e., $S \circ S=\mathrm{Id}$. Indeed, we have

$$
\mathbb{E}^{S \circ S(\mu)}[f(X)]=\mathbb{E}^{S(\mu)}[X f(1 / X)]=\mathbb{E}^{\mu}[(X / X) f(X)]=\mathbb{E}^{\mu}[f(X)]
$$


for all bounded measurable functions $f$. For future reference, we summarize our findings in the following lemma which also contains a few more properties, such as the fact that the operator $S$ preserves the convex order.

Lemma 2.3 The symmetry operator $S$ defined in (2.1) has the following properties:

(i) $S$ is an involution preserving the convex order in $\mathcal{P}_{1}$, i.e., $S \circ S=\mathrm{Id}$, and if $\mu, \nu \in \mathcal{P}_{1}$ satisfy $\mu \preccurlyeq v$, then $S(\mu) \preccurlyeq S(\nu)$.

(ii) If $\mu$ has density $p_{\mu}$, the measure $S(\mu)$ has a density given by $p_{S(\mu)}$ in (2.2).

(iii) If $\mu \in \mathcal{P}_{1}$, then for all $y>0$, we have

$$
F_{S(\mu)}(y)=1-G_{\mu}(1 / y) \quad \text { and } \quad G_{S(\mu)}(y)=1-F_{\mu}(1 / y) .
$$

Proof To prove property (i), it suffices to show that $S$ preserves the convex order of measures. Let $\mu, v \in \mathcal{P}_{1}$ be such that $\int f d \mu \leq \int f d v$ for any convex function $f$. Since $S(\mu)$ and $S(v)$ have both unit mass and the same first moment, it is enough to show that for any positive constants $K, L$ we have

$$
\mathbb{E}^{S(\mu)}\left[(K X-L)^{+}\right] \leq \mathbb{E}^{S(v)}\left[(K X-L)^{+}\right]
$$

Now $\mathbb{E}^{S(\mu)}\left[(K X-L)^{+}\right]=\mathbb{E}^{\mu}\left[X(K / X-L)^{+}\right]=\mathbb{E}^{\mu}\left[(K-L X)^{+}\right]$, and the same holds true for $v$. Since $x \mapsto(K-L X)^{+}$is a convex function, the result follows. Property (ii) has already been proved above, so it remains to show property (iii). We show only the left equality; the same arguments can be applied to get the other one. By the definition of $S$, we have

$$
\begin{aligned}
F_{S(\mu)}(y) & =S(\mu)(\{X \leq y\})=\mathbb{E}^{\mu}\left[X \mathbf{1}_{\{1 / X \leq y\}}\right] \\
& =\mathbb{E}^{\mu}[X]-\mathbb{E}^{\mu}\left[X \mathbf{1}_{\{X<1 / y\}}\right]=1-G_{\mu}(1 / y) .
\end{aligned}
$$

Hence the proof is complete.

\subsection{The symmetric two-marginals martingale transport problem}

In this subsection, we consider the change of numeraire in the two-period setting. Let $\mathbb{S}$ be the operator that assigns to every $Q \in \mathcal{M}(\mu, v)$ the measure $\mathbb{S}(Q)$ defined by

$$
\mathbb{E}^{\mathbb{S}(Q)}[f(X, Y)]=\mathbb{E}^{Q}\left[Y f\left(\frac{1}{X}, \frac{1}{Y}\right)\right] \text { for bounded measurable functions } f .
$$

Lemma 2.4 The operator $\mathbb{S}$ has the following properties:

(i) $\mathbb{S}(Q)$ is a probability in $\mathcal{M}(S(\mu), S(v))$ and satisfies $\mathbb{S} \circ \mathbb{S}=\mathrm{Id}$, i.e., $\mathbb{S}$ is an involution.

(ii) $\mathbb{S}(\mathcal{M}(\mu, v))=\mathcal{M}(S(\mu), S(v))$.

Proof (i) First, let us prove that $\mathbb{S}(Q) \in \mathcal{M}(S(\mu), S(\nu))$ for $Q \in \mathcal{M}(\mu, \nu)$. The fact that $Y$ has law $S(\nu)$ under $\mathbb{S}(Q)$ follows from the definition of $S$. Regarding $X$, by 
the martingale property under $Q$, we have

$$
\mathbb{E}^{\mathbb{S}(Q)}[f(X)]=\mathbb{E}^{Q}\left[Y f\left(\frac{1}{X}\right)\right]=\mathbb{E}^{Q}\left[X f\left(\frac{1}{X}\right)\right]
$$

for all bounded measurable functions $f$ depending only on $X$. Hence, using (2.1) yields that $X$ has law $S(\mu)$ under $\mathbb{S}(Q)$. It remains to show the martingale property under $\mathbb{S}(Q)$, and we first write

$$
\mathbb{E}^{\mathbb{S}(Q)}[Y f(X)]=\mathbb{E}^{Q}\left[Y \frac{1}{Y} f\left(\frac{1}{X}\right)\right]=\mathbb{E}^{Q}\left[f\left(\frac{1}{X}\right)\right]=\mathbb{E}^{Q}\left[X \frac{1}{X} f\left(\frac{1}{X}\right)\right] .
$$

Now by the martingale property under $Q$, we obtain $\mathbb{E}^{Q}\left[X \frac{1}{X} f\left(\frac{1}{X}\right)\right]=\mathbb{E}^{\mathbb{S}(Q)}[X f(X)]$, which implies $\mathbb{E}^{\mathbb{S}(Q)}[Y \mid X]=X$. The fact that $\mathbb{S}$ is an involution follows immediately from its definition.

(ii) In order to prove that $\mathbb{S}(\mathcal{M}(\mu, v))=\mathcal{M}(S(\mu), S(v))$, we note that one inclusion is implied by property (i). The other inclusion is a consequence of the fact that the symmetry operator $S$ is an involution.

Remark 2.5 Notice that the symmetry operator $S$ can be seen as the projection of $\mathbb{S}$. Indeed, the first part of the proof above gives that for any $Q \in \mathcal{M}(\mu, v)$ and all bounded measurable functions $f: \mathbb{R}_{++} \rightarrow \mathbb{R}$, we have $\mathbb{E}^{\mathbb{S}(Q)}[f(X)]=\mathbb{E}^{S(\mu)}[f(X)]$. In other words, the projection of $\mathbb{S}(Q)$ onto the first coordinate of the product space $\mathbb{R}_{++}^{2}$ equals $S(\mu)$. Similarly, one can see that the projection of $\mathbb{S}(Q)$ onto the second coordinate is $S(v)$.

Let $C: \mathbb{R}_{++}^{2} \rightarrow \mathbb{R}$ be any continuous function with linear growth, i.e., such that $|C(x, y)| \leq \kappa(1+x+y)$ for some constant $\kappa>0$. The lower and upper model-free price bounds for such a derivative can be computed by solving the martingale optimal transport problems

$$
\begin{aligned}
& \underline{P}(\mu, v, C):=\inf _{Q \in \mathcal{M}(\mu, v)} \mathbb{E}^{Q}[C(X, Y)], \\
& \bar{P}(\mu, \nu, C)=\sup _{Q \in \mathcal{M}(\mu, v)} \mathbb{E}^{Q}[C(X, Y)] .
\end{aligned}
$$

They have the interpretation of sub- and superreplication prices of the payoff $C$ through a duality theory that has been developed during the last few years by several authors (see Beiglböck et al. [2] and Beiglböck et al. [3], among others).

The following proposition shows the symmetry properties of such model-free bounds with respect to the change of numeraire transformation.

Proposition 2.6 Define the payoff $\mathbb{S}^{*}(C)(x, y):=y C\left(\frac{1}{x}, \frac{1}{y}\right)$ for $x, y>0$. Then

$$
\underline{P}\left(S(\mu), S(v), \mathbb{S}^{*}(C)\right)=\underline{P}(\mu, v, C), \quad \bar{P}\left(S(\mu), S(v), \mathbb{S}^{*}(C)\right)=\bar{P}(\mu, v, C) .
$$


Proof We only prove the equality for $\bar{P}$; the one for $\underline{P}$ can be shown using the same arguments. By definition of $\bar{P}$, we have

$$
\bar{P}\left(S(\mu), S(\nu), \mathbb{S}^{*}(C)\right)=\sup _{Q \in \mathcal{M}(S(\mu), S(v))} \mathbb{E}^{Q}\left[\mathbb{S}^{*}(C)(X, Y)\right] .
$$

Using property (ii) in Lemma 2.4 and the definition of $\mathbb{S}(Q)$, we get

$$
\begin{aligned}
\sup _{Q \in \mathcal{M}(S(\mu), S(v))} \mathbb{E}^{Q}\left[\mathbb{S}^{*}(C)(X, Y)\right] & =\sup _{Q \in \mathbb{S}(\mathcal{M}(\mu, v))} \mathbb{E}^{Q}\left[\mathbb{S}^{*}(C)(X, Y)\right] \\
& =\sup _{Q \in \mathcal{M}(\mu, v)} \mathbb{E}^{\mathbb{S}(Q)}\left[\mathbb{S}^{*}(C)(X, Y)\right] .
\end{aligned}
$$

Since $\mathbb{S}^{*}(C)=Y C(1 / X, 1 / Y)$ and by the definition of $\mathbb{S}(Q)$ in $(2.3)$, we obtain

$$
\mathbb{E}^{\mathbb{S}(Q)}\left[\mathbb{S}^{*}(C)(X, Y)\right]=\mathbb{E}^{\mathbb{S}(Q)}[Y C(1 / X, 1 / Y)]=\mathbb{E}^{Q}[C(X, Y)],
$$

yielding

$$
\bar{P}\left(S(\mu), S(\nu), \mathbb{S}^{*}(C)\right)=\sup _{Q \in \mathcal{M}(\mu, v)} \mathbb{E}^{Q}[C(X, Y)]=\bar{P}(\mu, v, C),
$$

which gives the result.

We conclude this section by showing how the symmetry operator $\mathbb{S}^{*}$ introduced in Proposition 2.6 acts on the space of hedgeable claims, which we define as

$$
\begin{aligned}
& \mathcal{H}(\mu, v)=\left\{C: \mathbb{R}_{++}^{2} \rightarrow \mathbb{R}: \text { there exist } \varphi \in \mathbb{L}^{1}(\mu), \psi \in \mathbb{L}^{1}(\nu), h \in m \mathcal{B}\left(\mathbb{R}_{++}\right),\right. \\
& \text {such that } \\
&C(x, y)=\varphi(x)+\psi(y)+h(x)(y-x) Q \text {-a.e., } \forall Q \in \mathcal{M}(\mu, \nu)\},
\end{aligned}
$$

where $m \mathcal{B}\left(\mathbb{R}_{++}\right)$stands for the space of all Borel-measurable real-valued functions defined on $\mathbb{R}_{++}$. This set contains all the payoffs that can be replicated by investing semi-statically in the stock as well as in vanilla options. It turns out that this set is invariant by the symmetry operator $\mathbb{S}^{*}$ or, in other words, the set of semi-static portfolios does not depend on the choice of the numeraire.

Proposition 2.7 The set $\mathcal{H}(\mu, v)$ is invariant by $\mathbb{S}^{*}$, in the sense that we have

$$
\mathbb{S}^{*}(\mathcal{H}(\mu, v))=\mathcal{H}(S(\mu), S(\nu)) .
$$

Proof First, we prove that $\mathbb{S}^{*}(\mathcal{H}(\mu, v)) \subset \mathcal{H}(S(\mu), S(v))$. Let $C \in \mathcal{H}(\mu, v)$, i.e., there exist functions $\varphi \in \mathbb{L}^{1}(\mu), \psi \in \mathbb{L}^{1}(\nu), h \in m \mathcal{B}\left(\mathbb{R}_{++}\right)$such that

$$
C(x, y)=\varphi(x)+\psi(y)+h(x)(y-x) \quad Q \text {-a.e., } \forall Q \in \mathcal{M}(\mu, v) .
$$

Let $\mathbb{S}^{*}(C)(x, y):=y C(1 / x, 1 / y)$ for all $x, y>0$ and let

$$
\tilde{\varphi}(x)=x \varphi(1 / x), \tilde{\psi}(y)=y \psi(1 / y), \tilde{h}(x)=(\varphi(1 / x)-1 / x h(1 / x)), \quad x, y>0 .
$$


By construction, these functions satisfy that $\tilde{\varphi} \in \mathbb{L}^{1}(S(\mu)), \tilde{\psi} \in \mathbb{L}^{1}(S(v))$ and $\tilde{h} \in m \mathcal{B}\left(\mathbb{R}_{++}\right)$. Moreover, one can check by direct computation that

$$
\mathbb{S}^{*}(C)(x, y)=\tilde{\varphi}(x)+\tilde{\psi}(y)+\tilde{h}(x)(y-x) \quad Q \text {-a.e., } \forall Q \in \mathcal{M}(\mu, v) .
$$

Now, since $\mathbb{S}(\mathcal{M}(\mu, v))=\mathcal{M}(S(\mu), S(\nu))$, we also have the equivalences

$$
\begin{gathered}
\mathbb{E}^{Q}[|C(X, Y)-\varphi(X)-\psi(Y)-h(X)(Y-X)|]=0, \forall Q \in \mathcal{M}(\mu, v) \\
\Longleftrightarrow \Longleftrightarrow \tilde{h}(X)(Y-X) \mid]=0, \forall Q \in \mathcal{M}(\mu, v) \\
\mathbb{E}^{\mathbb{S}(Q)}\left[\mid \mathbb{S}^{*}(C)(X, Y)-\tilde{\varphi}(X)-\tilde{\psi}(Y)-\tilde{h}\right) \\
\mathbb{E}^{Q}\left[\left|\mathbb{S}^{*}(C)(X, Y)-\tilde{\varphi}(X)-\tilde{\psi}(Y)-\tilde{h}(X)(Y-X)\right|\right]=0, \forall Q \in \mathcal{M}(S(\mu), S(v)) .
\end{gathered}
$$

As a consequence, we have

$$
\mathbb{S}^{*}(C)(x, y)=\tilde{\varphi}(x)+\tilde{\psi}(y)+\tilde{h}(x)(y-x) \quad Q \text {-a.e., } \forall Q \in \mathcal{M}(S(\mu), S(v)),
$$

i.e., $\mathbb{S}^{*}(C) \in \mathcal{H}(S(\mu), S(v))$. To prove the opposite inclusion, i.e.,

$$
\mathcal{H}(S(\mu), S(v)) \subset \mathbb{S}^{*}(\mathcal{H}(\mu, v)),
$$

we first observe that any $C \in \mathcal{H}(S(\mu), S(v))$ can be written as $C=\mathbb{S}^{*}(\tilde{C})$, where we define $\tilde{C}(x, y):=y C(1 / x, 1 / y)$. Hence the same arguments as in the first part of the proof (until (2.5)) apply and give $\tilde{C} \in \mathcal{H}(S \circ S(\mu), S \circ S(v))=\mathcal{H}(\mu, v)$ since $S$ is an involution. The proof is complete.

\section{Model-free pricing of forward start straddles}

In this section, we apply our results on the change of numeraire to compute the modelfree subreplication price of a forward start straddle of type I, which complements the result obtained in Hobson and Klimmek [10].

In their article, Hobson and Klimmek [10] consider the problem of computing a model-free lower bound on the price of an option paying $|Y-X|$ at maturity. This is an example of a type II forward start straddle, whose payoff for any strike $\alpha>0$ is given by

$$
C_{I I}^{\alpha}(x, y)=|y-\alpha x|, \quad x, y>0,
$$

while the type I forward start straddle with strike $\alpha>0$ is given by

$$
C_{I}^{\alpha}(x, y)=\left|\frac{y}{x}-\alpha\right|, \quad x, y>0 ;
$$

cf. Lucic [15] and Jacquier and Roome [11]. Hobson and Klimmek [10] derive explicit expressions for the coupling minimizing the model-free price of an at-themoney (ATM) type II forward start straddle $C_{I I}^{1}$ as well as for the corresponding subhedging strategy. In particular, they show that the optimal martingale coupling for such a derivative is concentrated on a three points transition $\{p(x), x, q(x)\}$, where 
$p$ and $q$ are two suitable decreasing functions. The precise result will be recalled below. Such a characterization is obtained under a dispersion assumption [10, Assumption 2.1] on the supports of the marginal laws: the support of $(\mu-v)^{+}$is contained in a finite interval $E$ and the support of $(v-\mu)^{+}$is contained in its complement $E^{c}$. Notice that the interval $E$ can be open, half-open or closed. Instead of working under such a condition on the supports, we rather impose the following standing assumption:

Assumption 3.1 Let the following properties hold:

(i) The measures $\mu$ and $v$ belong to $\mathcal{P}_{1}^{d}$.

(ii) $\delta F$ has a single local maximizer $m$.

The main reason for setting up this assumption is that it makes our proofs simpler and more uniform, without losing too much generality. Indeed, Assumption 3.1(i) implies that both marginals are atomless, which is the standing assumption used in the Henry-Labordère and Touzi [7] construction of the right- and left-monotone transference plans, which will be considered later in this paper. Moreover, in the case of marginals with densities, Assumption 3.1(ii) is equivalent to the dispersion assumption in [10] under the additional condition that $\mu$ and $v$ do not coincide on any subinterval of $\mathbb{R}_{++}$(as we show in the remark below). Notice that the latter condition is necessary for Assumption 3.1(ii) to hold.

Remark 3.2 Let $\mu, v \in \mathcal{P}_{1}^{d}$ with $\mu \preccurlyeq v$ and such that they do not coincide on any subinterval of $\mathbb{R}_{++}$. Then Assumption 2.1 in [10] is equivalent to our Assumption 3.1(ii). To see this, let $\mu, v \in \mathcal{P}_{1}$ with $\mu \preccurlyeq v$. Suppose that Assumption 2.1 in [10] holds, i.e., there exists a finite interval $E$ with endpoints $0 \leq a<b$ such that $\operatorname{supp}(\mu-v)^{+} \subset E$ and $\operatorname{supp}(v-\mu)^{+} \subset E^{c}$. Hence, by the definition of support, each $x>0$ such that $(\mu-v)((x-\epsilon, x+\epsilon))=\int_{(x-\epsilon, x+\epsilon)}\left(p_{\mu}(z)-p_{\nu}(z)\right) d z>0($ resp. $<0)$ for all $\epsilon>0$ satisfies $x \in E$ (resp. $x \in E^{c}$ ). Consequently, $\delta F$ is decreasing on $E$ and increasing on $E^{c}$. Hence, since $\mu$ and $v$ do not coincide on any sub-interval of $\mathbb{R}_{++}, \delta F$ admits a single local maximizer at $a$ and a single local minimizer at $b$, whence Assumption 3.1(ii) follows. Conversely, suppose that Assumption 3.1 holds. Then $\delta F$ has a single local maximizer $m>0$. Now, notice that $\delta F$ cannot be nonnegative over the whole half-line $\mathbb{R}_{++}$since also $\lim _{x \rightarrow 0} \delta F(x)=\lim _{x \rightarrow \infty} \delta F(x)=0$. Hence, by continuity, $\delta F$ has at least a global minimum at some point $\tilde{m}$. Moreover, the fact of having a single local maximizer implies that $\delta F$ cannot have more than one local minimizer. The convex order $\mu \preccurlyeq v$ is equivalent to $\int_{0}^{x} \delta F(z) d z \leq 0$ for all $x>0$, which implies $\tilde{m}<m$. Therefore, for almost every $x \in(\tilde{m}, m)$, we have $p_{\mu}(x)-p_{v}(x)>0$, while for almost every $x \in(\tilde{m}, m)^{c}$, we have $p_{\mu}(x)-p_{v}(x) \leq 0$, so that Assumption 2.1 in [10] is fulfilled.

Remark 3.3 Both properties in Assumption 3.1 are preserved under a change of numeraire. Indeed, we have already seen in Lemma 2.3 that $S(\mu), S(\nu)$ belong to $\mathcal{P}_{1}^{d}$. Concerning property (ii) in the assumption, note that

$$
F_{S(\mu)}(y)=\int_{0}^{y} \frac{p_{\mu}\left(\frac{1}{x}\right)}{x^{3}} d x=1-\int_{0}^{1 / y} x p_{\mu}(x) d x,
$$


so that

$$
\delta F_{S}(y)=F_{S(v)}-F_{S(\mu)}=-\int_{0}^{1 / y} x \partial_{x}(\delta F)(x) d x .
$$

Hence, $\delta F_{S}$ has a single local maximizer $x_{\star}^{S}$ if and only if $\delta F$ has a single local minimizer $x^{\star}$, satisfying $x^{\star}=\frac{1}{x_{\star}^{S}}$.

Let us come back to the model-free pricing of forward start straddles. Given the form of the payoff (3.1), it is very natural to try to obtain an optimal martingale coupling for its model-free subhedging price by combining the change of numeraire techniques with the Hobson and Klimmek [10] results. For the reader's convenience, we summarize their main result in the following theorem. It is a consequence of [10, Theorem 5.4 and 5.5] applied to the particular case when the marginals $\mu, v$ have densities (see their Sect. 6.1). Therefore, its proof is omitted.

Theorem 3.4 Let Assumption 3.1 hold. Then there exists a unique optimal coupling $\mathbb{Q}_{H K}(\mu, v)$ in $\mathcal{M}(\mu, v)$ such that

$$
\underline{P}\left(\mu, v, C_{I I}^{1}\right):=\inf _{Q \in \mathcal{M}(\mu, v)} \mathbb{E}^{Q}[|Y-X|]=\mathbb{E}^{\mathbb{Q}_{H K}(\mu, v)}[|Y-X|] .
$$

Moreover, $\mathbb{Q}_{H K}(\mu, v)(d x, d y)=\mu(d x) \mathcal{K}_{H K}(x, d y)$ with a transition kernel $\mathcal{K}_{H K}$ given by

$$
\begin{aligned}
\mathcal{K}_{H K}(x, \cdot)= & \delta_{x} \mathbf{1}_{\{x \leq a\}}+\left(\ell(x) \delta_{p(x)}+u(x) \delta_{q(x)}+(1-\ell(x)-u(x)) \delta_{x}\right) \mathbf{1}_{\{a<x<b\}} \\
& +\delta_{x} \mathbf{1}_{\{x \geq b\}},
\end{aligned}
$$

where

(i) a (resp. $b$ ) is the single local maximizer (resp. minimizer) of $\delta F$;

(ii) $p:(a, b) \rightarrow[0, a]$ and $q:(a, b) \rightarrow[b, \infty]$ are continuous decreasing functions which are solutions to the equations

$$
\begin{aligned}
& \delta F(q(x))+\delta F(p(x))=\delta F(x), \\
& \delta G(q(x))+\delta G(p(x))=\delta G(x), \quad x \in(a, b) ;
\end{aligned}
$$

(iii) $\ell, u:(a, b) \rightarrow[0,1]$ are given by

$$
\begin{aligned}
& u(x)=\frac{x-p(x)}{q(x)-p(x)} \frac{p_{\mu}(x)-p_{\nu}(x)}{p_{\mu}(x)}, \\
& \ell(x)=\frac{q(x)-x}{q(x)-p(x)} \frac{p_{\mu}(x)-p_{\nu}(x)}{p_{\mu}(x)} .
\end{aligned}
$$

Now, a simple application of the change of numeraire results from the previous section gives that $\mathbb{Q}_{H K}(\mu, v)$ attains the lower bound $\underline{P}(\mu, v, C)$ for the type I forward start straddle $C_{I}^{1}$ as well. This result complements the one in [10] about 
type II forward start straddle $C_{I I}^{1}$. We show first a symmetry property of the HobsonKlimmek optimal coupling.

Proposition 3.5 Let Assumption 3.1 hold. The martingale measure $\mathbb{Q}_{H K}(\mu, v)$ satisfies the symmetry relation

$$
\mathbb{S}\left(\mathbb{Q}_{H K}(S(\mu), S(v))\right)=\mathbb{Q}_{H K}(\mu, v),
$$

where the symmetry operator $\mathbb{S}$ is defined in (2.3).

Proof Let the pair $\left(p^{S}, q^{S}\right)$ define the measure $\mathbb{Q}_{H K}(S(\mu), S(v))$. A simple computation shows that the measure $\left.\mathbb{S}_{\left(\mathbb{Q}_{H}\right.}(S(\mu), S(\nu))\right)$ is concentrated on the set $\left\{\frac{1}{p^{S}(1 / x)}, x, \frac{1}{q^{S}(1 / x)}\right\}$. In order to get the equations satisfied by this three-band graph, recall first the symmetry relations

$$
\delta F^{S}(y)=-\delta G(1 / y), \quad \delta G^{S}(y)=-\delta F(1 / y) .
$$

By definition, $\left(p^{S}, q^{S}\right)$ is characterized by the two equations

$$
\begin{aligned}
& \delta F^{S}\left(q^{S}(x)\right)+\delta F^{S}\left(p^{S}(x)\right)=\delta F^{S}(x), \\
& \delta G^{S}\left(q^{S}(x)\right)+\delta G^{S}\left(p^{S}(x)\right)=\delta G^{S}(x) .
\end{aligned}
$$

Hence, using (3.2) we have

$$
\begin{aligned}
& \delta F\left(1 / q^{S}(1 / x)\right)+\delta F\left(1 / p^{S}(1 / x)\right)=\delta F(x), \\
& \delta G\left(1 / q^{S}(1 / x)\right)+\delta G\left(1 / p^{S}(1 / x)\right)=\delta G(x)
\end{aligned}
$$

Since the functions $x \mapsto 1 / p^{S}(1 / x)$ and $x \mapsto 1 / q^{S}(1 / x)$ are both continuous decreasing and satisfy the same equations as the pair $(p, q)$, they are candidates. Hence, the uniqueness of the optimal coupling yields the result.

At this point, we can exploit a symmetry relation between type I and type II forward start straddles, which is given by

$$
\mathbb{S}^{*}\left(C_{I I}^{\alpha}\right)(X, Y)=Y\left|\frac{1}{Y}-\frac{\alpha}{X}\right|=\alpha\left|\frac{Y}{X}-\frac{1}{\alpha}\right|=\alpha C_{I}^{\frac{1}{\alpha}} .
$$

In particular, the ATM straddles $(\alpha=1)$ are related by $\mathbb{S}^{*}\left(C_{I I}^{1}\right)(X, Y)=C_{I}^{1}(X, Y)$. Moreover, since $\mathbb{S}^{*}$ is an involution, we also have $\mathbb{S}^{*}\left(C_{I}^{1}\right)(X, Y)=C_{I I}^{1}(X, Y)$. A consequence of this is the following proposition, which states the announced result on forward start straddle of type I and concludes the section.

Proposition 3.6 Let Assumption 3.1 hold. The lower bound price of the ATM forward start straddle of type I is also attained by $\mathbb{Q}_{H K}(\mu, v)$, i.e.,

$$
\underline{P}\left(\mu, v, C_{I}^{1}\right):=\inf _{Q \in \mathcal{M}(\mu, v)} \mathbb{E}^{Q}\left[\left|\frac{Y}{X}-1\right|\right]=\mathbb{E}^{\mathbb{Q}_{H K}(\mu, v)}\left[C_{I}^{1}\right] .
$$


Proof Using Proposition 2.6 and the relation (3.3), we have

$$
\begin{aligned}
\underline{P}\left(\mu, v, C_{I}^{1}\right) & =\underline{P}\left(S(\mu), S(\nu), \mathbb{S}^{*}\left(C_{I}^{1}\right)\right)=\underline{P}\left(S(\mu), S(\nu), C_{I I}^{1}\right) \\
& =\mathbb{E}^{\mathbb{Q}_{H K}(S(\mu), S(v))}\left[C_{I I}^{1}\right]=\mathbb{E}^{\mathbb{Q}_{H K}(\mu, v)}\left[C_{I}^{1}\right],
\end{aligned}
$$

which ends the proof.

\section{Symmetry properties of left- and right-monotone transference plans}

The optimization problems in (2.4) are strongly related to the concepts of right-and left-monotone transference plans. Both notions were introduced in Beiglböck and Juillet [1], who show their existence and uniqueness for convex ordered marginals, and prove that they solve the maximization and the minimization problem in (2.4) for a specific set of payoffs of the form $C(x, y)=h(y-x)$ with $h$ differentiable with strictly convex first derivative. Henry-Labordère and Touzi [7] extend these results to a wider set of payoffs. Moreover, they also give an explicit construction of the leftmonotone transference plan. In this section, we study the symmetry property of those transference plans and show in particular that in the case of positive martingales, the right-monotone plan can be obtained from its left-monotone counterpart with no effort via a change of numeraire.

We start by recalling the general definition of right- and left-monotone transference plan.

Definition 4.1 A martingale measure $Q \in \mathcal{M}(\mu, v)$ is left-monotone (resp. rightmonotone) if there exists a Borel set $\Gamma \subset \mathbb{R}_{++}^{2}$ with $Q[\Gamma]=1$ such that for all $\left(x, y^{-}\right),\left(x, y^{+}\right)$and $\left(x^{\prime}, y^{\prime}\right)$ in $\Gamma$, we cannot have $x<x^{\prime}$ and $y^{-}<y^{\prime}<y^{+}$(resp. $x>x^{\prime}$ and $\left.y^{-}<y^{\prime}<y^{+}\right)$. We denote by $\mathbb{Q}_{L}(\mu, v)$ (resp. $\mathbb{Q}_{R}(\mu, v)$ ) the leftmonotone (resp. right-monotone) transference plan with marginals $\mu, \nu$.

The next result states how the two monotone transference plans relate to each other via the symmetry operators.

Proposition 4.2 The symmetry operator $\mathbb{S}$ exchanges left-monotone and right-monotone transference plans, i.e., we have the relations $\mathbb{S}\left(\mathbb{Q}_{R}(S(\mu), S(v))\right)=\mathbb{Q}_{L}(\mu, v)$ and $\mathbb{S}\left(\mathbb{Q}_{L}(S(\mu), S(\nu))\right)=\mathbb{Q}_{R}(\mu, v)$.

Proof We prove only the first equality as the second follows immediately since $\mathbb{S}$ is an involution. By definition of the right-monotone plan $Q_{R}^{S}:=\mathbb{Q}_{R}(S(\mu), S(v))$, there exists a Borel set $\Gamma_{R} \subset \mathbb{R}_{++}^{2}$ such that $Q_{R}^{S}\left[\Gamma_{R}\right]=1$ and for all $\left(x, y^{-}\right),\left(x, y^{+}\right)$, $\left(x^{\prime}, y^{\prime}\right)$ in $\Gamma_{R}$, we cannot have $x>x^{\prime}$ and $y^{-}<y^{\prime}<y^{+}$. Let

$$
\Gamma_{R}^{\mathbb{S}}:=\left\{(x, y) \in \mathbb{R}_{++}^{2}:(1 / x, 1 / y) \in \Gamma_{R}\right\} .
$$

We clearly have

$$
\mathbb{S}\left(Q_{R}^{S}\right)\left[\Gamma_{R}^{\mathbb{S}}\right]=\mathbb{E}^{Q_{R}^{S}}\left[Y \mathbf{1}_{\Gamma_{R}^{\mathbb{S}}}(1 / X, 1 / Y)\right]=\mathbb{E}^{Q_{R}^{S}}\left[Y \mathbf{1}_{\Gamma_{R}}(X, Y)\right]=\mathbb{E}^{Q_{R}^{S}}[Y]=1
$$


Since $\left(x, y^{-}\right),\left(x, y^{+}\right),\left(x^{\prime}, y^{\prime}\right)$ are in $\Gamma_{R}^{\mathbb{S}}$ if and only $\left(1 / x, 1 / y^{-}\right),\left(1 / x, 1 / y^{+}\right)$, $\left(1 / x^{\prime}, 1 / y^{\prime}\right)$ are in $\Gamma_{R}$, we cannot have $x<x^{\prime}$ and $y^{-}<y^{\prime}<y^{+}$. Moreover, we have $\mathbb{S}\left(\mathbb{Q}_{R}(S(\mu), S(\nu))\right) \in \mathcal{M}(\mu, \nu)$; hence by uniqueness of the left-monotone transference plan (see [1, Theorem 1.5]), we obtain $\mathbb{S}\left(Q_{R}^{S}\right)=\mathbb{Q}_{L}(\mu, v)$.

Remark 4.3 We observe that as a by-product of the previous result, the existence of a left-monotone transference plan for marginal laws $\mu, v$ gives for free the existence of its right-monotone analogue, but for a different pair of marginals $S(\mu), S(\nu)$, via the symmetry operator $\mathbb{S}$ and vice versa. Moreover, notice also that the result above holds in full generality, i.e., even when the marginals do not have densities.

Building on the results in [1], Henry-Labordère and Touzi [7] show in particular that $\mathbb{Q}_{L}(\mu, v)$ attains the upper bound (2.4) for a larger class of payoffs satisfying a generalized Spence-Mirrlees type condition $C_{x y y}>0$ (or $C_{x y y}<0$ ) (see their Theorem 5.1). We summarize their result in the following theorem. ${ }^{1}$

Theorem 4.4 (Henry-Labordère and Touzi [7]) Let $C: \mathbb{R}_{++}^{2} \rightarrow \mathbb{R}$ be a measurable function such that the partial derivative $C_{x y y}$ exists and $C_{x y y}>0$. Under Assumption 3.1, the left-monotone transference plan $Q_{L}=Q_{L}(\mu, v)$ is the optimal coupling solving the martingale transport problem

$$
\bar{P}(\mu, \nu, C):=\sup _{Q \in \mathcal{M}(\mu, v)} \mathbb{E}^{Q}[C(X, Y)] .
$$

In order to apply the change of numeraire approach, notice first that by the definition of $\mathbb{S}^{*}(C)$, we have

$$
\mathbb{S}^{*}(C)_{x y y}(x, y)=-\frac{1}{x^{2} y^{3}} C_{x y y}\left(\frac{1}{x}, \frac{1}{y}\right), \quad x, y>0 .
$$

Hence, we have that $C_{x y y}>0$ holds true if and only if $\mathbb{S}^{*}(C)_{x y y}<0$. This elementary remark allows us to find the model-free price bounds for payoffs satisfying $C_{x y y}<0$ by changing the numeraire. This is similar to what happens with the mirror coupling in [7, Remark 5.2], where the marginals have support in $\mathbb{R}$. The symmetry operators $S$ and $\mathbb{S}$ permit to handle this case for $\mathbb{R}_{++}$-supported marginals.

To make this observation more precise, let $C(x, y)$ be a payoff satisfying $C_{x y y}<0$. Hence $\mathbb{S}^{*}(C)_{x y y}>0$ and by Proposition 2.6, we have

$$
\begin{aligned}
\bar{P}(\mu, v, C) & =\bar{P}\left(S(\mu), S(v), \mathbb{S}^{*}(C)\right) \\
& =\mathbb{E}^{\mathbb{Q}_{L}(S(\mu), S(v))}\left[\mathbb{S}^{*}(C)(X, Y)\right] \\
& =\mathbb{E}^{\mathbb{S}\left(\mathbb{Q}_{L}(S(\mu), S(v))\right)}[C(X, Y)] .
\end{aligned}
$$

Therefore, $\bar{P}(\mu, v, C)$ is attained by $\left.\mathbb{S}_{\left(\mathbb{Q}_{L}\right.}(S(\mu), S(v))\right)$, which is equal to $\mathbb{Q}_{R}(\mu, v)$ by Proposition 4.2. One can prove in a similar way that if $C_{x y y}>0$ (resp. $\left.C_{x y y}<0\right)$, the lower bound in $(2.4)$ is attained by $\mathbb{Q}_{R}(\mu, v)\left(\operatorname{resp} . \mathbb{Q}_{L}(\mu, v)\right)$.

\footnotetext{
${ }^{1}$ Observe that the results in Theorem 4.4 hold under more general conditions than our Assumption 3.1(ii).
} 
Remark 4.5 We say that a payoff function $C$ is symmetric if it satisfies $\mathbb{S}^{*}(C)=C$. $^{2}$ For any symmetric payoff $C$ which satisfies the slightly relaxed generalized SpenceMirrlees condition $C_{x y y} \geq 0$, we can use (4.1) to get $C_{x y y}(x, y)=-\frac{1}{x^{2} y^{3}} C_{x y y}\left(\frac{1}{x}, \frac{1}{y}\right)$, hence $C_{x y y}=0$. Integrating twice with respect to $y$ and once with respect to $x$, we see that $C$ is necessarily of the form $C(x, y)=\varphi(x)+\psi(y)+h(x)(y-x)$ for some functions $\varphi, \psi$ and $h$.

\subsection{Explicit constructions of left and right-monotone transference plans and change of numeraire}

In this section, we briefly recall the explicit construction of a left-monotone transference plan performed in [7] and show how the change of numeraire can be used to generate, essentially for free, the basic right-monotone transport plan from its leftmonotone counterpart via the symmetry operator. We stress that Assumption 3.1 is still in force. The explicit characterization of $Q_{L}$ in [7] is described, for the reader's convenience, in the following theorem.

Theorem 4.6 Let Assumption 3.1 hold. The left-monotone transference plan $Q_{L}$ is given by $Q_{L}(d x, d y)=\mu(d x) \mathcal{K}_{L}(x, d y)$ with transition kernel

$$
\mathcal{K}_{L}(x, \cdot)=\delta_{x} \mathbf{1}_{\left\{x \leq x_{\star}\right\}}+\left(q_{L}(x) \delta_{L_{u}(x)}+\left(1-q_{L}(x)\right) \delta_{L_{d}(x)}\right) \mathbf{1}_{\left\{x>x^{\star}\right\}},
$$

where $q_{L}(x):=\frac{x-L_{d}(x)}{L_{u}(x)-L_{d}(x)}, x_{\star} \in \mathbb{R}_{++}$is the unique maximizer of $\delta F$, and $L_{d}, L_{u}$ are positive continuous functions on $(0, \infty)$ such that

(i) $L_{d}(x)=L_{u}(x)=x$ for $x \leq x_{\star}$;

(ii) $L_{d}(x)<x<L_{u}(x)$ for $x>x_{\star}$;

(iii) on the interval $\left(x^{\star}, \infty\right), L_{d}$ is decreasing and $L_{u}$ is increasing.

Moreover, $L_{d}$ is the unique solution to

$$
F_{v}^{-1}\left(F_{\mu}(x)+\delta F\left(L_{d}(x)\right)\right)=G_{v}^{-1}\left(G_{\mu}(x)+\delta G\left(L_{d}(x)\right)\right), \quad x>x_{\star},
$$

and $L_{u}$ is given by the relation

$$
F_{v}\left(L_{u}(x)\right)=F_{\mu}(x)+\delta F\left(L_{d}(x)\right), \quad x>x_{\star} .
$$

Proof We refer to [7, Theorem 4.5]. More details on the case of a single maximizer can be found in Sect. 3.4 therein.

Now, using the fact that $\mathbb{S}\left(Q_{L}(S(\mu), S(v))\right)=Q_{R}(\mu, v)$ together with the characterization of the left-monotone transference plan given in the previous theorem, we can investigate how the quantities defining $Q_{R}$ and $Q_{L}$ are related to each other. Notice that since both marginals have support in $\mathbb{R}_{++}$, the symmetry relation we use here is different from the one in [7, Remark 5.2].

\footnotetext{
${ }^{2} \mathrm{~A}$ way of constructing a symmetric payoff $C$ goes as follows: choose its values on $[0,1] \times \mathbb{R}_{++}$first, then for $(x, y) \in(1, \infty) \times \mathbb{R}_{++}$, set $C(x, y)=y C(1 / x, 1 / y)$. One can easily check that $C$ satisfies $\mathbb{S}^{*}(C)=C$.
} 
Proposition 4.7 Let Assumption 3.1 hold. Then the right-monotone transference plan $Q_{R}$ is given by $Q_{R}(d x, d y)=\mu(d x) \mathcal{K}_{R}(x, d y)$ with transition kernel

$$
\mathcal{K}_{R}(x, \cdot):=\delta_{x} \mathbf{1}_{\left\{x \leq x^{\star}\right\}}+\left(q_{R}(x) \delta_{R_{u}(x)}+\left(1-q_{R}(x)\right) \delta_{R_{d}(x)}\right) \mathbf{1}_{\left\{x>x^{\star}\right\}},
$$

where

(i) $x^{\star}=1 / x_{\star}^{S}$ is the unique minimizer of $\delta F$;

(ii) $R_{d}(x)=\frac{1}{L_{u}^{S}(1 / x)}$ and $R_{u}(x)=\frac{1}{L_{d}^{S}(1 / x)}$ for $x>0$;

(iii) the transition probability is given by $q_{R}(x)=\frac{x}{R_{u}(x)}\left(1-q_{L}^{S}(1 / x)\right)$ for $x>0$.

Proof By Lemma 2.3, if $\mu, v \in \mathcal{P}_{1}$ satisfy $\mu \preccurlyeq v$, then their images by the symmetry operator $S$ satisfy the same conditions, i.e., $S(\mu), S(\nu) \in \mathcal{P}_{1}$ and $S(\mu) \preccurlyeq S(\nu)$. By Remark 3.3, one has that $\delta F_{S}=F_{S(v)}-F_{S(\mu)}$ has a single local maximizer, and Theorem 4.6 gives that there exists a left-monotone transference plan $Q_{L}^{S}:=Q_{L}(S(\mu), S(\nu))$ characterized as in Theorem 4.6.

To conclude, since we already know that $\mathbb{S}\left(Q_{L}^{S}\right)=Q_{R}(\mu, v)$ (see Proposition 4.2), it suffices to check that the measure $\tilde{Q}$ defined as $\tilde{Q}(d x, d y):=\mu(d x) \mathcal{L}_{R}(d x, d y)$ with the kernel $\mathcal{L}_{R}$ defined as in the statement satisfies

$$
\mathbb{E}^{\tilde{Q}}[f(X, Y)]=\mathbb{E}^{\mathbb{S}\left(Q_{L}^{S}\right)}[f(X, Y)]
$$

for all bounded measurable functions $f:\left(\mathbb{R}_{++}\right)^{2} \rightarrow \mathbb{R}$. This can be done by direct computation using the formulas for $x^{\star}, R_{d}$ and $R_{u}$ given in the statement. The details are therefore omitted.

Remark 4.8 As a by-product of the previous proposition, we get the characterization of $Q_{R}$ in terms of a triplet $\left(x_{\star}, R_{d}, R_{u}\right)$, where $x_{\star}>0$ is the unique minimizer of $\delta F$ and $R_{d}, R_{u}$ are positive continuous functions on $\mathbb{R}_{++}$which solve

$$
\begin{gathered}
F_{\nu}^{-1}\left(F_{\mu}(x)+\delta F\left(R_{u}(x)\right)\right)=G_{v}^{-1}\left(G_{\mu}(x)+\delta G\left(R_{u}(x)\right)\right), \\
G_{\nu}\left(R_{d}(x)\right)-G_{\mu}(x)=G_{\nu}\left(R_{u}(x)\right)-G_{\mu}\left(R_{u}(x)\right) .
\end{gathered}
$$

\section{Summary}

In this paper, we have introduced change of numeraire techniques in the twomarginals transport problem for positive martingales. In particular, we have studied the symmetry properties of the Hobson and Klimmek [10] optimal coupling under the change of numeraire, which exchanges type I with type II forward start straddles. As a consequence, we have proved that the lower bound prices are attained for both options by the Hobson-Klimmek transference plan. On the other hand, relying on the construction of Henry-Labordère and Touzi [7] of the optimal transference plan introduced by Beiglböck and Juillet [1], we have also shown that the change of numeraire transformation exchanges the left- and the right-monotone transference plans, so that the latter can be viewed as a mirror coupling acting on the former under a change of numeraire for positive martingales with given marginals. 
Acknowledgements This work is partially supported by the ANR project ISOTACE (ANR-12-MONU0013). We are also grateful to Stefano De Marco, Pierre Henry-Labordère, David Hobson and Antoine Jacquier for their helpful remarks.

Open Access This article is distributed under the terms of the Creative Commons Attribution 4.0 International License (http://creativecommons.org/licenses/by/4.0/), which permits unrestricted use, distribution, and reproduction in any medium, provided you give appropriate credit to the original author(s) and the source, provide a link to the Creative Commons license, and indicate if changes were made.

\section{References}

1. Beiglböck, M., Juillet, N.: On a problem of optimal transport under marginal martingale constraints. Ann. Probab. 44, 42-106 (2016)

2. Beiglböck, M., Henry-Labordère, P., Penkner, F.: Model-independent bounds for option pricesa mass transport approach. Finance Stoch. 17, 477-501 (2013)

3. Beiglböck, M., Nutz, M., Touzi, N.: Complete duality for martingale optimal transport on the line. Ann. Probab. (2016, forthcoming). arXiv:1507.00671

4. Dolinsky, Y., Soner, H.M.: Martingale optimal transport and robust hedging in continuous time. Probab. Theory Relat. Fields 160, 391-427 (2014)

5. Dolinsky, Y., Soner, H.M.: Martingale optimal transport in the Skorokhod space. Stoch. Process. Appl. 125, 3893-3931 (2015)

6. Geman, H., El Karoui, N., Rochet, J.-C.: Changes of numeraire, changes of probability measure and option pricing. J. Appl. Probab. 32, 443-458 (1995)

7. Henry-Labordère, P., Touzi, N.: An explicit martingale version of Brenier's theorem. Finance Stoch. 20, 635-668 (2016)

8. Hobson, D.: Robust hedging of the lookback option. Finance Stoch. 2, 329-347 (1998)

9. Hobson, D.: The Skorokhod embedding problem and model-independent bounds for option prices. In: Carmona, R.A., et al. (eds.) Paris-Princeton Lectures on Mathematical Finance 2010. Lecture Notes in Mathematics, vol. 2003, pp. 267-318. Springer, Berlin (2011)

10. Hobson, D., Klimmek, M.: Robust price bounds for the forward starting straddle. Finance Stoch. 19, 189-214 (2015)

11. Jacquier, A., Roome, P.: Asymptotics of forward implied volatility. SIAM J. Financ. Math. 6, 307-351 (2015)

12. Jamshidian, F.: An exact bond option formula. J. Finance 44, 205-209 (1989)

13. Jeanblanc, M., Yor, M., Chesney, M.: Mathematical Methods for Financial Markets. Springer, Berlin (2009)

14. Laachir, I.: Quantification of the model risk in finance and related problems. Ph.D. thesis, EnstaParisTech (2015). Available online at https://tel.archives-ouvertes.fr/tel-01305545/document

15. Lucic, V.: Forward-start options in stochastic volatility models. In: Wilmott, P. (ed.) The Best of Wilmott 1: Incorporating the Quantitative Finance Review, pp. 413-420. Wilmott Magazine (2004)

16. Strassen, V.: The existence of probability measures with given marginals. Ann. Math. Stat. 36, 423439 (1965) 